\title{
Contrôle actif de vibrations
}

\author{
Valérie Pommier ${ }^{1}$ - Marc Budinger ${ }^{2}$ - Bernard Mouton ${ }^{3}$ - Sylvaine Leleu ${ }^{4}$ \\ ${ }^{1,3}$ ENSICA (Ecole Nationale Supérieure d'Ingénieurs de Constructions Aéronautiques) Toulouse, vpommier@ensica.fr \\ ${ }^{2}$ Lycée Champollion, Figeac, marc budinger@yahoo.com \\ ${ }^{4}$ Lycée Polyvalent Jean Jaurès, 25 rue Charles Lecoq, 95100 Argenteuil, sylvaine.leleu@,wanadoo.fr
}

\begin{abstract}
RESUME : Cet article présente un exemple de maquette de contrôle actif à partir de céramiques piézoélectriques. Cette maquette fait l'objet d'un projet à l'ENSICA au cours duquel les étudiants doivent mettre en œuvre leurs connaissances en automatique et en électronique. Ce projet permet aux étudiants de mettre en place une chaine de mesure, de concevoir et réaliser des circuits d'électronique et enfin de calculer et implémenter une loi de commande.
\end{abstract}

Mots clés : automatique, électronique, contrôle actif, céramiques piézoélectriques, chaînes de mesures, loi de commande

\section{INTRODUCTION}

Dans beaucoup de cas, les vibrations représentent des nuisances. Elles sont sources de bruit acoustique et de détérioration du matériel. Le contrôle de vibrations consiste à diminuer l'amplitude des vibrations d'une structure qui vibre. Deux familles de solutions sont envisageables :

- le contrôle passif de vibrations avec des matériaux qui les amortissent (par exemple des matériaux viscoélastiques)

- le contrôle actif avec des actionneurs qui sont mis en mouvement pour venir contrer ces vibrations. Cette solution nécessite un apport d'énergie pour la mise en mouvement des actionneurs mais elle peut s'avérer plus efficace dans certains cas.

Cet article présente un projet sur le contrôle actif de vibrations utilisant des céramiques piézoélectriques. Ce projet permet d'illustrer des notions fondamentales en électronique et en automatique. Il est partagé en six séances de 2,5 heures qui se présentent sous forme de cours, de simulations avec Matlab et de réalisation pratique.

\section{LES MATERIAUX}

\section{PIEZOELECTRIQUES [1]}

\subsection{L'effet piézoélectrique}

La piézoélectricité (voir figure 1) est la propriété que possèdent certains corps de se polariser électriquement sous l'action d'un effort mécanique (effet direct) et de se déformer lorsqu'un champ électrique leur est appliqué (effet inverse).

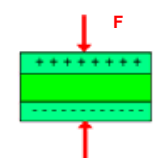

Figure 1 - Effet piézo-électrique

Le couplage entre la direction du champ électrique et la direction de déformation peut se faire selon plusieurs directions que l'on peut classer selon trois modes principaux : longitudinal (33), transversal (31) et de cisaillement (51).
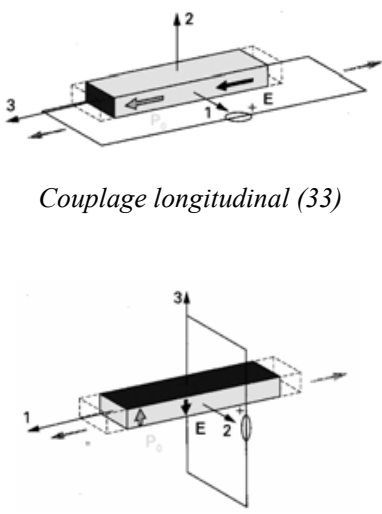

Couplage transversal (31)

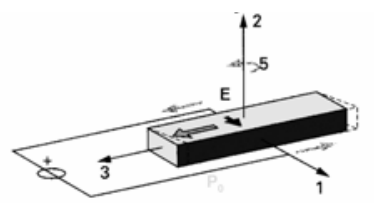

Couplage de cisaillement (51)

Figure 2-Modes de couplages [2] 


\subsection{Les matériaux piézoélectriques PZT}

Les matériaux piézoélectriques utilisés couramment sont les céramiques PZT (figure 3) (plomb, zirconium et titane).

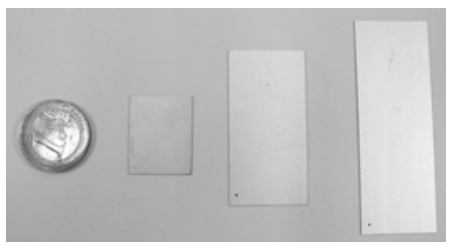

Figure 3 - Céramiques PZT

On distingue deux grandes familles de céramiques PZT :

- Les céramiques douces qui se déforment bien pour une tension d'alimentation donnée. Mais leurs caractéristiques peuvent se dégrader fortement pour des contraintes et des fréquences élevées. Elles sont principalement utilisée en micro positionnement (statique ou basse fréquence) et en capteurs.

- Les céramiques dures qui se déforment moins pour une tension d'alimentation donnée mais qui conservent leurs caractéristiques aux hautes fréquences et pour des fortes contraintes. Elles ont également moins de pertes électriques et mécaniques. Elles sont utilisées pour les applications à la résonance et des applications qui nécessitent de la puissance.

\subsection{Les matériaux piézoélectriques PVDF}

On trouve aussi des matériaux PVDF (Figure 4) qui se présentent sous forme de films et présentent l'avantage d'être souples et donc de s'adapter à des surfaces non planes. Ils sont plutôt utilisés comme capteurs.

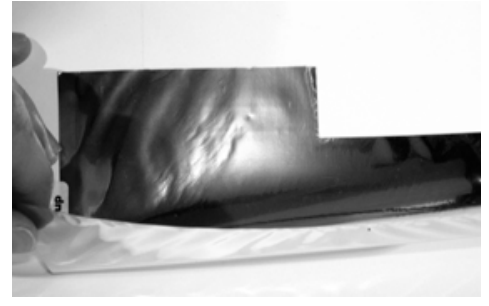

Figure 4 - Film PVDF

\section{ETUDE D'UNE MAQUETTE POUR}

\section{LE CONTROLE ACTIF DE}

\section{VIBRATIONS}

La maquette décrite ci-après est inspirée de la thèse de Sylvaine Leleu menée au laboratoire du SATIE de l'ENS de Cachan [3].

\subsection{Description de la maquette}

La maquette constitue un exemple simple de contrôle actif de vibrations: une simple poutre encastrée-libre équipée :

- d'un actionneur piézoélectrique de type céramique PZT (P151 de PI Polytech [4]) qui peut générer des vibrations qui vont modérer les vibrations perturbatrices de façon à obtenir une diminution globale des vibrations mécaniques sur la structure.

- d'un capteur piézoélectrique de type PVDF (PVDF mono-orienté métallisé de Piezotech) qui permet de mesurer les vibrations de la structure.

L'actionneur et le capteur fonctionnent en mode 31. Ils sont placés au niveau de l'encastrement car c'est la position où l'actionneur aura le plus d'effet et où le capteur donnera le plus de signal (voir Figure 5).
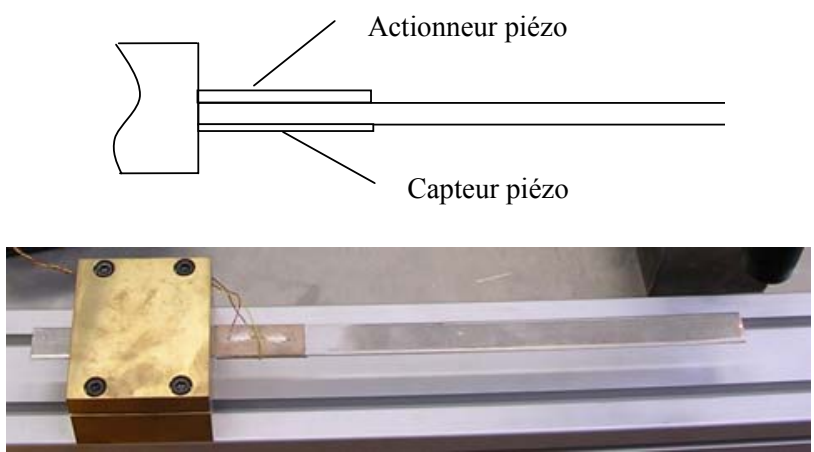

Figure 5 - Exemple simple de structure de contrôle actif de vibrations

L'actionneur piézoélectrique est alimenté par une alimentation de $\pm 130 \mathrm{~V}$ via un amplificateur de tension

Le capteur piézoélectrique sensible aux déformations mécaniques génère une variation de charges électriques. Afin de convertir ce signal en une tension, qui est une grandeur plus facilement exploitable, on utilise un amplificateur de charges [5]. Le signal issu de l'amplificateur est une image des vibrations dans la poutre. Ce signal est traité de manière analogique ou numérique afin de calculer la tension à envoyer à l'actionneur afin de diminuer les vibrations dans la structure. Il s'agit donc d'un système asservi.

Le schéma complet de la maquette est représenté dans la figure 6 . 


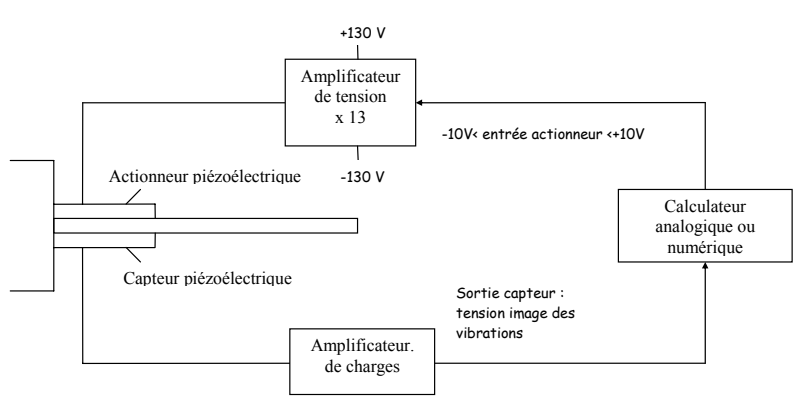

Figure 6-Schéma complet de la maquette de contrôle actif

\subsection{Descriptif des différents éléments de la maquette}

La poutre

La structure de base est une poutre encastrée-libre en aluminium. Ses caractéristiques mécaniques et géométriques sont données dans la figure 7 et le tableau 1.

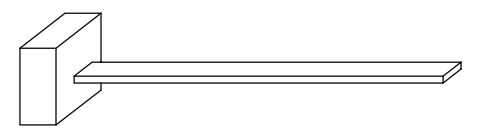

Figure 7 - Poutre encastrée

\begin{tabular}{|l|c|}
\hline Longueur L & $300 \mathrm{~mm}$ \\
\hline Largeur 1 & $20 \mathrm{~mm}$ \\
\hline Epaisseur e & $2 \mathrm{~mm}$ \\
\hline Module d'Young E & $70 \mathrm{Gpa}$ \\
\hline Masse Volumique $\varphi$ & $2970 \mathrm{~kg} / \mathrm{m}^{3}$ \\
\hline
\end{tabular}

Tableau 1 - Tableau des caractéristiques

En théorie, les fréquences propres pour une poutre encastrée-libre sont données par la formule :

$$
f_{i}=\lambda_{i}^{2} \sqrt{\frac{E I}{\varphi \cdot l \cdot e}} * \frac{1}{2 \cdot \pi \cdot L^{2}},
$$

avec $I=$ Moment quadratique $=1 * \mathrm{e}^{3} / 12$

et $\lambda_{\mathrm{i}}$ un coefficient relatif à chacun des modes calculé à partir de la géométrie de la structure et des conditions aux limites [6].

En appliquant la formule précédente les différentes fréquences propres obtenues sont :

\begin{tabular}{|c|c|c|}
\hline Modes & $\boldsymbol{\lambda}_{\mathbf{i}}$ & $\mathbf{f}_{\mathbf{i}}$ \\
\hline Mode 1 & 1.875 & $18.3 \mathrm{~Hz}$ \\
\hline Mode 2 & 4.694 & $114.5 \mathrm{~Hz}$ \\
\hline Mode 3 & 7.854 & $320.7 \mathrm{~Hz}$ \\
\hline Mode 4 & 10.995 & $628.5 \mathrm{~Hz}$ \\
\hline
\end{tabular}

Tableau 2 : Valeurs des fréquences propres

\section{L'amplificateur de tension}

Pour obtenir une déformation significative de la poutre, on alimente les actionneurs piézoélectriques avec une tension de $\pm 130 \mathrm{~V}$ à leurs bornes. Le signal de commande atteignant $\pm 10 \mathrm{~V}$ au maximum, l'amplificateur doit avoir un gain de 13 pour alimenter les actionneurs en $\pm 130 \mathrm{~V}$. Cette tension de sortie est obtenue grâce à un amplificateur de puissance de chez APEX 'Référence PB58' (voir Figure 8).

L'amplificateur "PB58" ou "Booster" se situe sur le $2^{\text {ème }}$ étage d'amplification. Au premier étage d'amplification, on a un simple Amplificateur de type TL071 appelé 'Driver'.

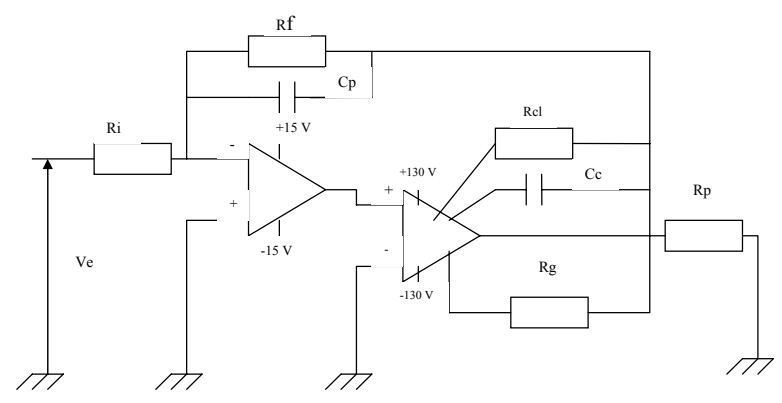

Figure 8-Schéma de l'amplificateur de tension

\section{L'amplificateur de charges}

Le capteur est relié à un amplificateur de charges dont le schéma est donné ci-dessous (Figure 9).

Le principe de cet amplificateur est d'imposer, via l'amplificateur opérationnel, une différence de potentiel nulle entre les deux électrodes du capteur, afin d'annuler l'influence des capacités (câblage ou capteur). Les charges sont redirigées vers la capacité $C_{a}$ aux bornes de laquelle il suffit de mesurer la tension afin de déterminer la quantité de charges. La résistance en parallèle $R_{a} a$ pour effet d'effectuer un filtrage passe haut afin d'éliminer les éventuelles charges parasites basse fréquence.

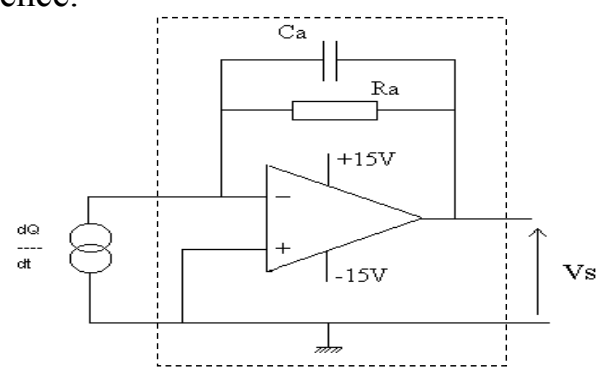

Figure 9-Amplificateur de charges

La fonction de transfert de l'amplificateur de charges a pour expression :

$$
V_{S}=-\frac{1}{p C_{a}+\frac{1}{R_{a}}} * \frac{d Q}{d t}
$$


soit

$$
\frac{V_{S}}{Q}=-\frac{1}{C_{a}} * \frac{R_{a} C_{a} p}{1+R_{a} C_{a} p}=-\frac{1}{C_{a}} * \frac{\tau * p}{1+\tau * p}
$$

avec $\tau=\mathrm{R}_{\mathrm{a}} \mathrm{C}_{\mathrm{a}}$.

Le diagramme de Bode en gain d'une telle fonction est donné dans la figure 10 .

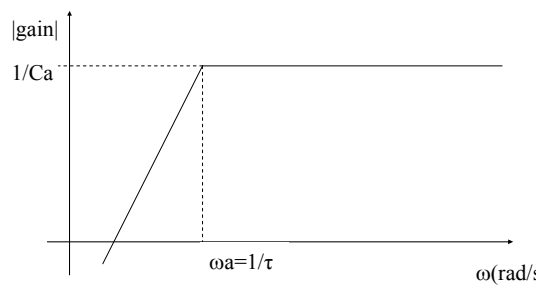

Figure 10 - Diagramme de Bode en gain de l'amplificateur de charges

Pour dimensionner l'amplificateur de charges, on se base sur la première fréquence propre qui est de $18.3 \mathrm{~Hz}$. On choisit $\omega_{\mathrm{a}} \approx \omega_{1} / 10$ avec $\omega_{1}=2 \pi \mathrm{f}_{1}$ de manière à ne supprimer aucune fréquence propre de la poutre. On prend la résistance $R_{a}$ la plus grande possible de façon à ce que le condensateur $\mathrm{C}_{\mathrm{a}}$ soit le plus possible petit. Cela permet d'avoir un gain de l'amplificateur grand afin de maximiser le rapport signal/bruit.

\begin{tabular}{|c|c|}
\hline composants & valeurs \\
\hline $\mathrm{Ca}$ & $8 \mathrm{nF}$ \\
\hline $\mathrm{Ra}$ & $10 \mathrm{M} \Omega$ \\
\hline $\mathrm{fa}$ & $2 \mathrm{~Hz}$ \\
\hline $\mathrm{f1}$ & $18.3 \mathrm{~Hz}$ \\
\hline
\end{tabular}

Tableau 3 - Choix des composants pour l'amplificateur de charges

\subsection{Modèle de la poutre active}

On modélise la structure comme une somme de systèmes du second ordre mais on s'intéresse uniquement au premier mode. On recherche donc un modèle du premier mode sous la forme :

$$
T(p)=\frac{K}{\frac{p^{2}}{\omega_{p}{ }^{2}}+\frac{2 \xi p}{\omega_{p}}+1}
$$

avec :

- $\omega_{\mathrm{p}}$ la pulsation propre qui est ici proche de la pulsation de résonance

- $K$ le gain statique de la fonction de transfert

- $\xi$ l'amortissement qui se déduit du coefficient de qualité grâce à la formule : $Q=\frac{1}{2 \xi}$

Des mesures effectuées sur la maquette permettent d'évaluer les paramètres du modèle. A partir du diagramme de Bode du système «amplificateur de tension + poutre + amplificateur de charges » (Figure 11), on mesure :

- les fréquences de résonance réelles du système (tableau 4). La légère différence observée entre les valeurs pratiques des fréquences propres et les valeurs théoriques de la formule du paragraphe 2.2 provient du fait que les capteurs et actionneurs piézoélectriques ne sont pas pris en compte dans cette formule.

- Le coefficient de qualité $Q$ de la structure active pour le premier mode en utilisant les fréquences à $-3 \mathrm{~dB}$ (Figure 12). On trouve $Q=93$.

- le gain statique entre le signal d'entrée et le signal de sortie. On trouve un gain de 0.05.

Toutes ces informations conduisent à la fonction de transfert suivante :

$$
T(p)=\frac{0.05}{\left(\frac{p}{125}\right)^{2}+\frac{0.0107}{125} p+1}
$$

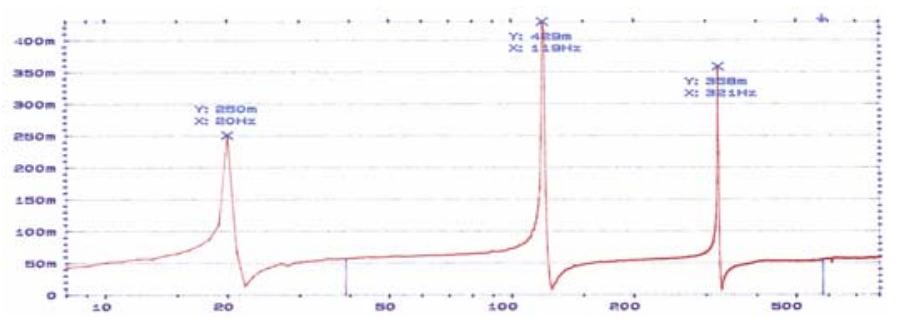

Figure 11 - Diagramme de Bode de la poutre (abscisse=fréquence $(\mathrm{Hz})$, Ordonnée $y=$ Gain)

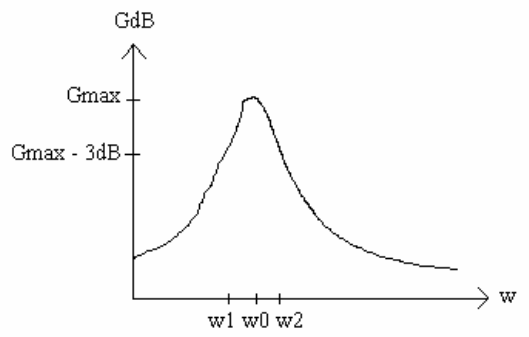

Facteur de qualité: $Q=\omega_{0} /\left(\omega_{2}-\omega_{1}\right)$

Figure 12 - Mesure du facteur de qualité

\begin{tabular}{|c|c|c|}
\hline Modes & $\mathrm{f}_{\mathrm{i}}$ (pratique) & $\mathrm{f}_{\mathrm{i}}$ (théorique) \\
\hline Mode 1 & $20 \mathrm{~Hz}$ & $18,3 \mathrm{~Hz}$ \\
\hline Mode 2 & $119 \mathrm{~Hz}$ & $114,5 \mathrm{~Hz}$ \\
\hline Mode 3 & $321 \mathrm{~Hz}$ & $320,7 \mathrm{~Hz}$ \\
\hline Mode 4 & $605 \mathrm{~Hz}$ & $628,5 \mathrm{~Hz}$ \\
\hline
\end{tabular}

Tableau 4 - Valeurs pratiques et théoriques des différentes fréquences propres 


\subsection{Calcul de la loi de commande}

La méthode de correction choisie est simple et rapide : le Positive Position Feedback ou 'PPF', Elle peut s'appliquer car l'actionneur et le capteur sont co-localisés. Ce correcteur 'PPF' est assimilé à un filtre du $2^{\text {ème }}$ ordre. Il est placé dans la chaîne de retour de la boucle, avec une contreréaction positive. Le schéma de commande est représenté dans la figure 13.

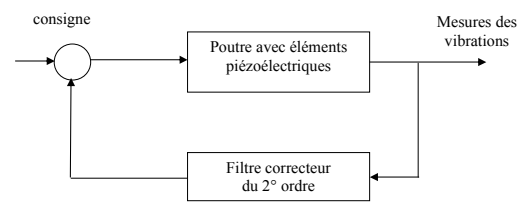

Figure 13 - Schéma de commande avec PPF

La correction est ici réalisée pour le mode 1 uniquement, par l'insertion d'un filtre du second ordre du type :

$$
C_{P P F}(p)=\frac{G_{f_{1}} \omega_{f 1}^{2}}{p^{2}+2 \varsigma_{f 1} \omega_{f 1} p+\omega_{f 1}^{2}}
$$

La pulsation propre du filtre est choisie telle que $\omega_{\mathrm{fl}}=1,3 \omega_{1}$ et que son amortissement soit environ égal à 0,7 . Le gain $\mathrm{G}_{\mathrm{f} 1}$ est calculé de manière à obtenir un signal de sortie amorti pour le mieux, sans saturer l'actionneur.

On peut observer les simulations des diagrammes de Bode de la poutre seule et de la poutre équipée d'un système PPF (Figure 14). On remarque bien le pic de résonance sans contrôle et l'effet du PPF qui augmente fortement l'amortissement du système.

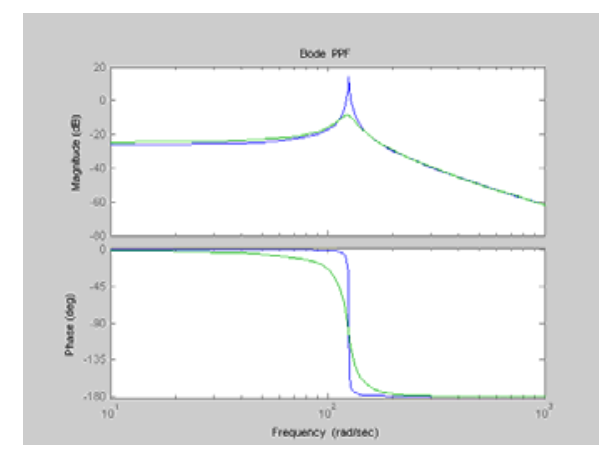

Figure 14 - Diagrammes de Bode de la poutre sans et avec PPF

\subsection{Résultats d'essais}

Deux solutions sont possibles pour implanter la loi de commande.

- En analogique, on peut réaliser le correcteur $C_{P P F}$ à partir d'un schéma de filtre de Rauch ou de Sallen-Key.

- En numérique, on peut implanter le correcteur dans une carte de commande. Dans notre cas, le banc est équipé d'une carte d'acquisition avec un DSP qui vient faire l'interface entre l'ordinateur et la poutre (Figure 15). Grâce au programme Matlab compilé et chargé dans le DSP, la commande à imposer à l'amplificateur est calculée en temps réel puis envoyée à l'actionneur.

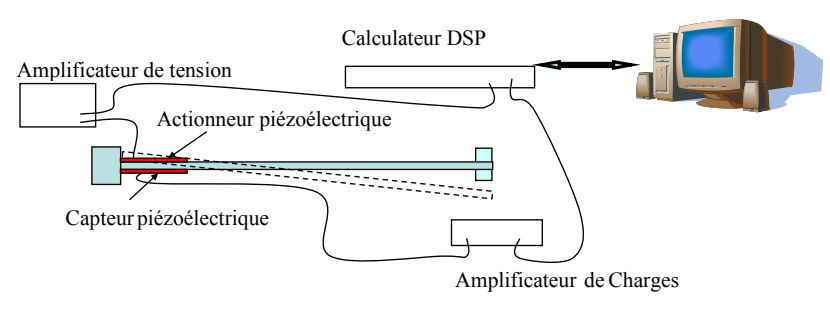

Figure 15 - Montage du système asservi

Des essais de lâcher, qui excitent essentiellement le premier mode, sont réalisés. Les résultats de ces essais sont donnés dans les figures 16 et 17 (base de temps de la figure $16: 500 \mathrm{~ms}$ et base de temps de la figure 17: 100ms). La poutre est bien amortie. On peut comparer la rapidité de correction et l'amortissement pour une excitation initiale correspondant à une flèche en bout de poutre de $8 \mathrm{~mm}$ (sans vitesse initiale). Sans contrôle, l'amortissement vaut 0.01 et les vibrations sont amorties au bout de plus de 4 secondes. Avec le contrôle PPF, l'amortissement vaut 0.08 et les vibrations sont amorties au bout de 0.7 seconde environ. 


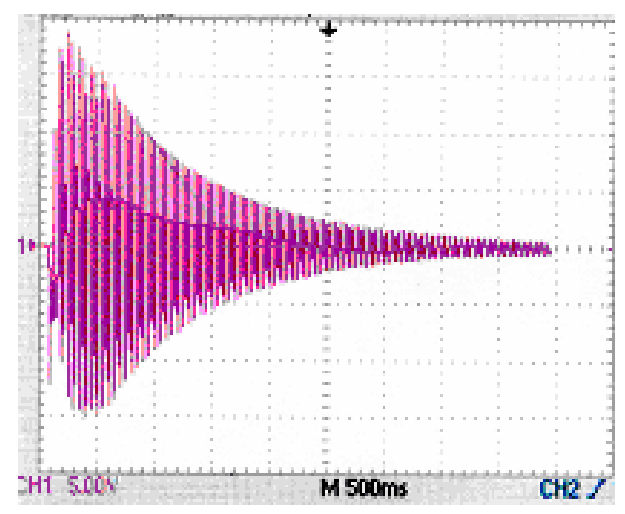

Figure 16-Réponse sans contrôle actif à une excitation en bout de poutre

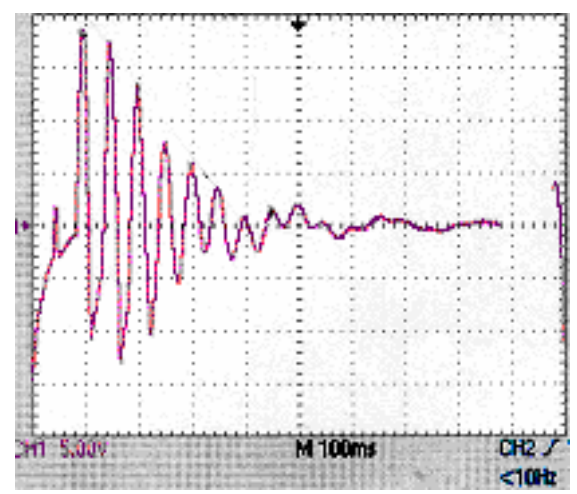

Figure 17 - Réponse avec contrôle actif à une excitation en bout de poutre

\section{DEROULEMENT DU PROJET}

Le projet issu de cette maquette se déroule en 5 séances de 2.5 heures chacune :

- Séance 1: Présentation du contrôle actif, des matériaux piézoélectriques et des différents éléments de la maquette.

- Séance 2: Réalisation en électronique analogique de l'amplificateur de charges. Réglage du gain à partir de la notion de signal sur bruit. Mesures pour déterminer un modèle de la poutre active.

- Séance 3: Calcul de la loi de commande à partir du modèle établi en séance 2 avec Matlab.

- Séances 4 et 5 : Réalisation de la loi de commande en analogique à partir d'une structure de Rauch ou de Sallen-Key puis en numérique avec la carte de commande avec le DSP. Introduction à la notion de robustesse en automatique: on modifie la structure avec une masse en bout de poutre et on observe le comportement du système asservi dans les différentes configurations.

\section{CONCLUSION}

Un exemple de maquette de contrôle actif à partir de céramiques piézoélectriques a été présenté. Ce projet permet aux étudiants de mettre en œuvre leurs connaissances en automatique et en électronique. Ils doivent :

- mettre en place une chaîne de mesure

- concevoir et réaliser des circuits d'électronique

- calculer et implémenter une loi de commande

\section{Bibliographie :}

1 Marc Budinger, «Contribution à la conception et à la modélisation d'actionneurs piézoélectriques cylindriques à deux degrés de liberté de type rotation et translation ", Thèse de l'INP de Toulouse, 2003

2 Bertrand Nogarède, "Moteurs piézoélectriques », Techniques de l'ingénieur, Traité Génie électrique, D3765, juillet 1996

3 Sylvaine Leleu, "Amortissement actif des vibrations d'une structure flexible de type plaque à l'aide de transducteurs piézoélectriques", Thèse du SATIE de l'ENS Cachan, 2002

4 www.polytec-pi.fr/

5 Georges Asch, "Les capteurs en instrumentation industrielle", Ed. Dunod, 1998

6 Robert D. Blevins, "Formulas for Natural Frequency and Mode Shape", Ed. Hardcover, 2001 